\title{
Contribuições ao estudo da anatomia macroscópica do encéfalo do Bradypus torquatus (Linnaeus, 1758) e Bradypus variegatus (Schinz, 1825)
}

Jussara Rocha FERREIRA ${ }^{1}$ Vera Lúcia de OLIVEIRA² Kleber Mirallia de OLIVEIRA ${ }^{3}$

Nozelmar Borges de SOUSA-JÚNIOR ${ }^{3}$

\section{Correspondência para:}

JUSSARAROCHAFERREIRA Instituto de Ciências Biológicas Universidade Federal de Goiás Rua 111,250 - Setor Sul 74085-130 - Goiânia - GO mirallia@bol.com.br

Recebido para publicação: 29/05/2003 Aprovado para publicação: 13/07/2005

\author{
1- Instituto de Ciências Biológicas da Universidade Federal de Goiás, \\ Goiânia - GO \\ 2- Projeto de preservação do bicho preguiça da reserva Zoobotânica \\ (Matinha) de Ilhéus - CEPLAC, Ilhéus - BA \\ 3- Universidade Salgado de Oliveira, Goiânia - GO
}

\section{Resumo \\ A grande preocupação na preservação da fauna sul americana tem levado pesquisadores a intensa produção de trabalhos. Este trabalho} constitui parte deste esforço para entender as estruturas animais, em especial do gênero Bradypus, ordem Edentata, descrevendo a anatomia macroscópica do encéfalo de duas espécies, Torquatus e Variegatus, ambas ocorrendo na mata atlântica brasileira. Os animais foram a óbito naturalmente, congelados reserva da Matinha, e descongelados, injetados, dissecados e corados por de técnicas apropriadas. Vemos as formações do neocórtex num modelo mais primitivo para mamíferos, uma predominância de áreas e estruturas ligadas ao olfato e diversas diferenças, quando comparadas a outros mamíferos, em especial a primatas. Dentre estas, a ocorrência de apenas 2 colículos no tronco encefálico, hipófise discoidal, grande fórnice e bem desenvolvidos pedúnculos cerebrais. As circunvoluções são brandas e ocorrem lobos semelhantes aos demais mamíferos, com cerebelo não recoberto pelas projeções dos telencéfalos e com proporção de até $1 / 4$ em relação ao todo.

\section{Introdução}

Há na sociedade contemporânea uma crescente preocupação com a preservação da natureza e, conseqüentemente, com as diversas formas de vida animal do continente Sul-Americano que têm sido objeto de estudo em pesquisas recentes tratando de variados temas: o sistema digestivo em lhamas ${ }^{1}$, em cutias ${ }^{2}$, em primatas ${ }^{3,4}$; o sistema circulatório em cutias $^{5}$, em pacas ${ }^{6}$, em catetos $^{7}$, o aparelho reprodutor em pacas ${ }^{8}$; o sistema nervoso em capivaras ${ }^{9}$ e de primatas $^{10,11,12}$. Vários aspectos da biologia, forma e função do preguiça (Bradypus) foram estudados ${ }^{13}$, da anatomia externa do telencéfalo ${ }^{14}$ e da constituição do plexo lombo sacral ${ }^{15}$.

Isto demonstra que os pesquisadores brasileiros estão preocupados e empenhados em produzir conhecimentos para que, através da pesquisa científica, se possa contribuir com a manutenção destas vidas, considerando que dos cerca de 3000 gêneros conhecidos de mamíferos, 2000 já foram extintos $^{16}$. Entre os mamíferos das 16 ordens de Eutheria existentes, destaca-se um grupo intrigante de animais, os do gênero Edentata, cujos representantes, no continente sulamericano, são os tamanduás, os tatus e os preguiças.

Com relação a este último grupo animal, nos propusemos a desenvolver estudos sobre aspectos da morfologia das preguiças (Bradypus) em parceria com a CEPLAC 2002 (Comissão Executiva do Plano da Lavoura Cacaueira) - CEPEC (Centro de Pesquisas do Cacau), através do Projeto de Preservação do Bicho Preguiça. Este estudo se caracteriza como uma 
abordagem inicial da anatomia do encéfalo em Bradypus torquatus (Bt) e Bradypus variegatus $(\mathrm{Bv})$, utilizando encéfalos dos quais se retirou a dura-máter, se executou uma análise externa e se preparou cortes longitudinais e frontais para estudo e docência, uma vez que um dos objetivos do projeto de preservação do preguiça inclui a educação ambiental.

\section{Materiais e Métodos}

Utilizamos, neste trabalho, os encéfalos de quatro animais do gênero Bradypus (torquatus - Bt e variegatus - Bv), sendo dois de adultos e um filhote Bt e apenas um adulto $\mathrm{Bv}$. Os animais vieram a óbito naturalmente na Reserva Zôo-botânica (Matinha) de Ilhéus/Itabuma, no estado da Bahia, localizada em área sob a responsabilidade da CEPLAC (Comissão Executiva do Plano da Lavoura Cacaueira). Foram cedidos para projetos de pesquisa em parceria entre a Reserva de Matinha, a Faculdade de Medicina Veterinária e Zootecnia da Universidade de São Paulo e o Instituto de Ciências Biológicas da Universidade Federal de Goiás.

Após o óbito, os animais foram congelados como rotina na Reserva Biológica. Procedeu-se descongelamento natural (temperatura ambiente, 24 horas), perfusão com água aquecida $\left(40^{\circ} \mathrm{C}\right)$ via aorta torácica; injeção com solução aquosa de formol a $10 \%$ para fixação. Retirou-se os encéfalos, utilizando uma lupa óptica da marca LTS modelo 3700, após o que procedeu-se a remoção das meninges em uma lupa estereoscópica da marca Zeiss, modelo Stemi 2000-C com aumento de 100 vezes. Um dos encéfalos (adulto, $\mathrm{Bt}$ ) foi fatiado em cortes coronais (Figura 1H), fotografado e a seguir corado pela técnica de Mullingan ${ }^{17}$; com a finalidade de documentar a topografia das substâncias cinzenta e branca e servir como referência para estudos. $O$ encéfalo de outro espécime (filhote de Bt) teve seu encéfalo fatiado em secção sagital mediana (Figura 1E). Nos outros animais, (adultos $\mathrm{Bt}$ e $\mathrm{Bv}$ ) identificamos outras características de macroscopia externa do encéfalo. Utilizamos a nomenclatura anatômica veterinária oficial ${ }^{18}$ e a nomenclatura anatômica veterinária ilustrada ${ }^{19}$, para denominações das estruturas identificadas.

\section{Resultados e Discussão}

Analisando as partes do sistema nervoso central do $\mathrm{Bt}$ e $\mathrm{Bv}$ verificamos que como em outros animais descritos ${ }^{16}$, o encéfalo e a medula espinhal (somente segmento C1) se apresentam como estruturas contínuas sem delimitação evidente (Figura 1B). O cérebro é relativamente irregular e suas circunvoluções estão recobertas pelos folhetos das meninges e ossos do crânio.

O tamanho do encéfalo correspondeu a uma média de 5,5 centímetros de comprimento, 3 centímetros de largura e 2,2 centímetros de altura, tendo o cérebro fixado pesado em média 16 gramas (Tabela 1), não há dados de encéfalos não fixados para obtermos um índice de correção aplicável à variação do peso deste animal. Do mesmo modo, o número de animais é insuficiente para uma análise estatística apropriada, esperamos que ao adicionarmos outros dados, inclusive de outros grupos de pesquisa, isto seja possível.

O desenvolvimento relativo de algumas partes do encéfalo, em particular, demonstrou uma preponderância de áreas de neocórtex e neocerebelo, mais recentemente adquiridas no desenvolvimento filogenético (Figuras $1 \mathrm{Fe} \mathrm{G}$ ), ao mesmo tempo observou-se um cérebro olfativo muito significativo (Figuras $1 \mathrm{~B} \mathrm{e} \mathrm{F}$ ), no qual os tractos e os lobos olfativos são bem desenvolvidos, Jollie ${ }^{20}$ afirma que este animais usam do olfato para sua vida social. $\mathrm{O}$ aumento de volume e de complexidade do telencéfalo ficou evidente, tanto em relação aos adultos da mesma espécie (Bt), quanto ao da outra (Bv) (Figuras 1C e F).

É fato bem estabelecido que filhotes têm cérebros menos circunvolutos que os adultos, nas figuras $1 \mathrm{~A}, \mathrm{C} \mathrm{e} \mathrm{F}$, a primeira de 


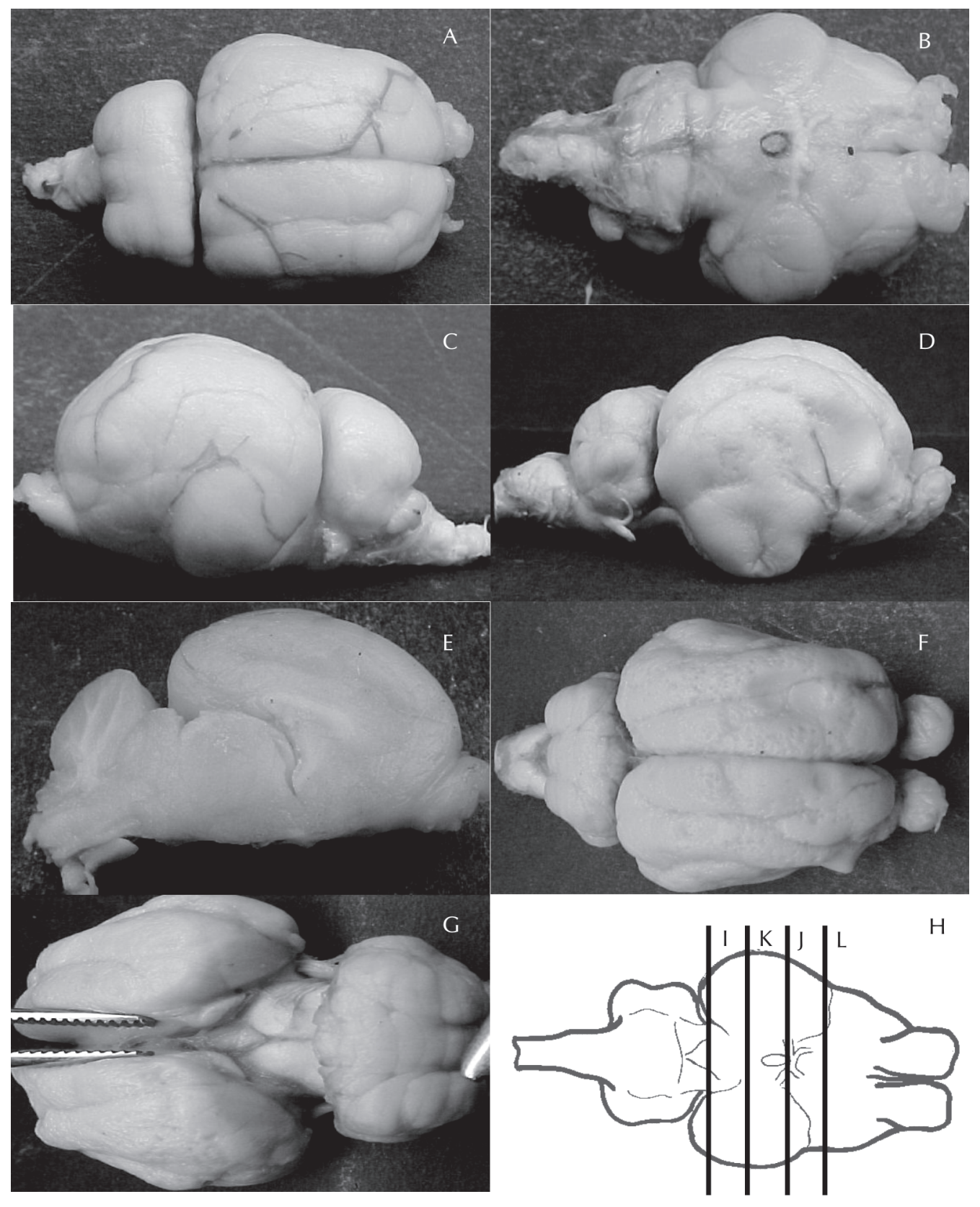

Figura 1 - Fotografias do encéfalo de animal jovem, Bradypus torquatus (Bt), em vista dorsal (A), ventral (B), lateral esquerda (C), lateral direita (D), secção sagital mediana (E); e de animal adulto, Bradypus variegatus (Bv) em vista dorsal (F), dorsal com afastamento do cerebelo G); esquema de cortes em $(\mathrm{H})$. Os números se referem às estruturas do sistema nervoso central indicadas na tabela 2 

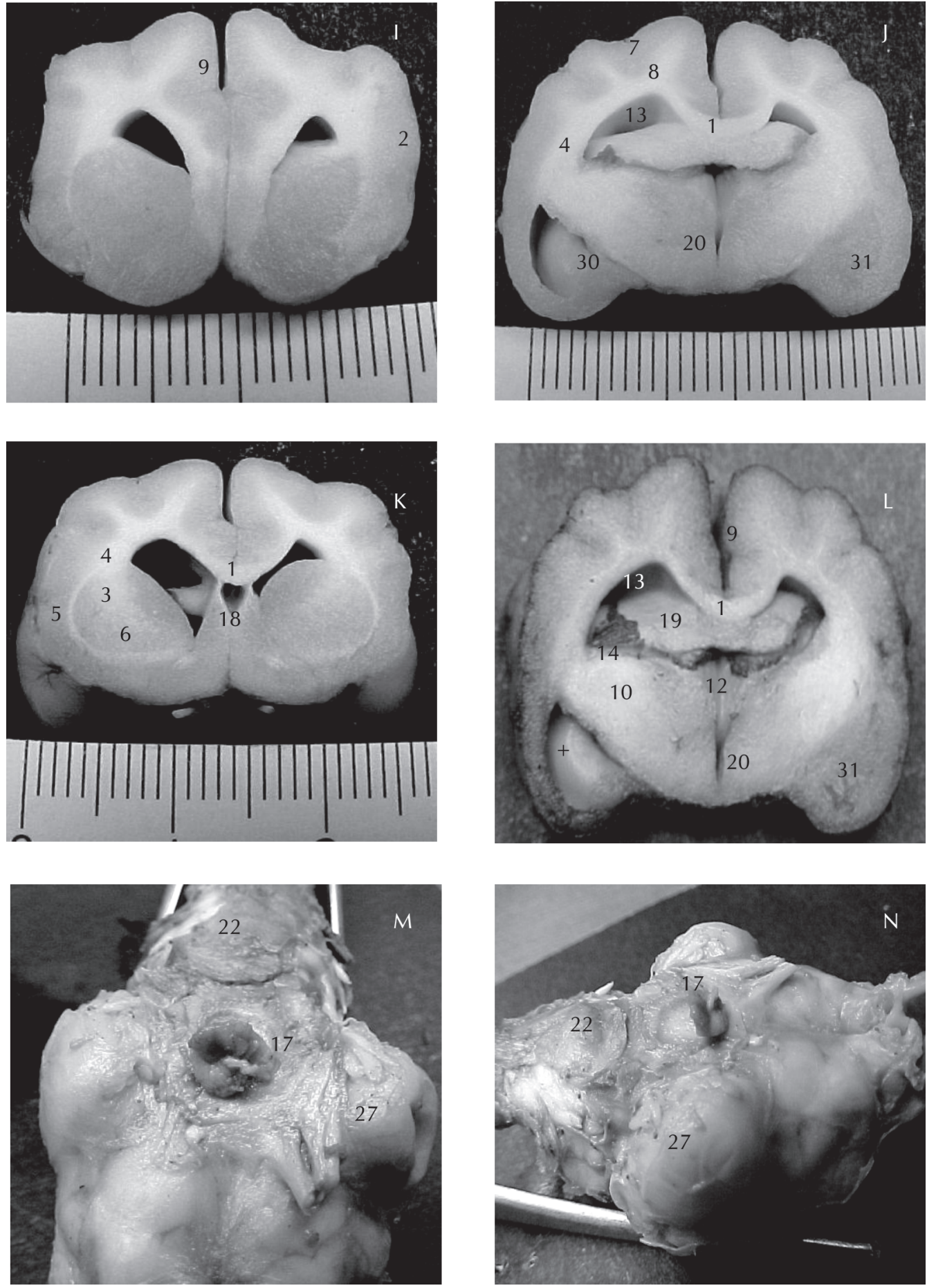

Figura 2 - Fotografias de secções frontais do encéfalo de Bradypus torquatus, (I - secção ,J, K, L) e de vista ventral e ventro-lateral de Bradypus variegatus $(\mathrm{M}, \mathrm{N})$. Numeração refere-se à tabela 1. ( + - córtex insular); ( $\downarrow$-infundíbulo da hipófise) 
Tabela 1 - Dimensõese dados dos animais utilizados, médias dos adultos

\begin{tabular}{cccccc}
\hline Animais & 1 & 2 & 3 & Média & Filhote \\
\hline Peso(gr) & 16.4 & 15 & 17 & 16 & 7 \\
\hline Largura(cm) & 3 & 2,8 & 3,2 & 3 & 2 \\
\hline Espessura(cm) & 2,6 & 2 & 2 & 2,2 & 1,8 \\
\hline Comprimento(cm) & 5.7 & 5 & 6 & 5,5 & 3,7 \\
\hline Sexo & Masc. & Fem. & Masc. & - & Masc. \\
\hline Idade & $\sim 2$ anos & $\sim 3$ anos & $\sim 2,5$ anos & - & 4 meses \\
\hline Espécie & Bt & Bt & Bv & - & Bt
\end{tabular}

um recém nascido e a última de um animal adulto, o encéfalo, como em outros mamíferos superiores, se modificou com a idade. Observa-se no animal jovem as circunvoluções menos evidenciadas. As imagens (Figuras 1D, E e F) nos permitiram considerar que os Bradypus têm um cerebelo relativamente desenvolvido em relação a répteis e anfíbios, também pode-se notar na vista ventral (Figura 1B) o correspondente volume da região pontina que acomoda os sistemas de fibras ascendentes e descendentes destinados ao cerebelo ${ }^{21}$. Quanto aos hemisférios cerebrais, estes são semi-ovais com uma evidente expansão postero-lateral no lobo temporal (Figuras 1A, D e F). Os hemisférios cerebrais vistos pela sua superfície cranial são divididos entre si por uma fissura longitudinal mediana e estão separados do cerebelo por uma fissura transversal praticamente retilínea no filhote de Bt (Figura 1A) e ligeiramente abaulada na linha mediana em sentido caudo-rostral no adulto do $\mathrm{Bv}$ (Figura 1F).

Os hemisférios cerebrais, em ambos os antímeros, exibem circunvoluções bastante pronunciadas, num formato de giro particularmente mais largo e alongado em relação a caninos e felinos. Embora Dyce, Sack e Wensing ${ }^{22}$, Frandson e Spurgeon ${ }^{23}$, Hildelbrand ${ }^{16}, \mathrm{Hill}^{24}$, Jollie ${ }^{20}$ tenham documentado em esquemas, encéfalos de vários vertebrados superiores, estes, macroscopicamente, não se aproximaram muito das representações que registramos nos bichos preguiças. Se não forem consideradas as circunvoluções, a morfologia do encéfalo deste animal se aproximou mais à representação feita para o eqüino conforme documentação de Nusshag ${ }^{25}$. A face ventral do encéfalo é plana e apresentou como em outros mamíferos a emergência aparente dos nervos cranianos. A parte mais caudal do encéfalo é composta pela medula oblonga, vindo em direção rostral a ponte com cerca do dobro da largura, não ficou evidente a presença do sulco bulbo-pontino (Figura 1B). Dorsalmente à ponte, encontra-se o cerebelo. Os hemisférios cerebrais estão conectados por meio do corpo caloso sem identificarmos comissuras dorsal ou rostral, contudo é possível identificarmos a adesão inter-talâmica e a comissura do fórnice (Figura 2, J, K, L); os mesmos se conectam ao cerebelo e tronco encefálico por meio dos pedúnculos cerebrais (o encéfalo médio) que se apresentam como duas colunas divergentes projetadas caudo-rostralmente cobertas pelo cerebelo na face dorsal, por sob os hemisférios cerebrais (Figura 1G) sem apresentar a fossa interpeduncular. Há a presença neste sítio de uma protuberância ventral, o hipotálamo ao qual se liga a glândula hipófise, alojada na face ventral da fossa craniana media e tendo esta o formato discoidal, presa ao encéfalo por um pedúnculo localizado em sua parte rostral.(Figuras $2 \mathrm{M}$ e $\mathrm{N}$ ).

As secções frontais e sagitais nos permitiram identificar no $\mathrm{Bv}$ e $\mathrm{Bt}$ os elementos anatômicos relacionados na tabela 1 e indicados nas imagens constantes das figuras 1 e 2 . Chamamos a atenção para a 
Tabela 2 - Legenda das estruturas identificadas na analise dosencéfalos e ocorrência das mesmas nas figuras 1 e 2 . Os números se referem a legenda das figuras

\begin{tabular}{|c|c|c|c|}
\hline Nome da estrutura & $\begin{array}{l}\text { Identificação numérica no } \\
\text { texto e nas figuras. }\end{array}$ & Figura 1 & Figura 2 \\
\hline Corpo caloso & 1 & $\mathrm{E}, \mathrm{G}$. & K, L. \\
\hline Hemisférios cerebrais & 2 & $A, D, F$ & \\
\hline Coroa radiada & 3 & & $\mathrm{~K}, \mathrm{~J}$ \\
\hline Substância branca sub-cortical & 4 & & $\mathrm{~K}, \mathrm{~L}$. \\
\hline Cápsula Externa & 5 & & K. \\
\hline Corpo estriado & 6 & & $\mathrm{I}, \mathrm{K}$ \\
\hline $\begin{array}{l}\text { Córtex cerebral (substância } \\
\text { cinzenta) }\end{array}$ & 7 & & J, L. \\
\hline Substância branca & 8 & & J, K, L. \\
\hline Fissura longitudinal mediana & 9 & G & I, J, K, L. \\
\hline Tálamo & 10 & $\mathrm{E}$ & $\mathrm{L}$. \\
\hline Pedúnculo cerebral & 11 & G & $\mathrm{L}$. \\
\hline $3^{\circ}$ ventrículo & 12 & $E$ & J, L. \\
\hline Ventrículo lateral & 13 & & I, J, K, L. \\
\hline Plexo coróide & 14 & & $\mathrm{~L}$. \\
\hline Colículos & 15 & G & \\
\hline Bulbo olfatório & 16 & $A, B, C, D, E, F$ & \\
\hline Hipófise & 17 & & $\mathrm{M}, \mathrm{N}$. \\
\hline Septo pelúcido e sua cavidade & 18 & & $\mathrm{~K}$. \\
\hline Fórnice & 19 & $E$ & J, L. \\
\hline $3^{\circ}$ ventrículo (note o aqueduto) & 20 & $\mathrm{E}$ & J, L. \\
\hline Corpo pineal & 21 & G & \\
\hline Ponte & 22 & $\mathrm{~B}, \mathrm{E}$ & \\
\hline $\begin{array}{l}\text { Cerebelo, hemisférios direito e } \\
\text { esquerdo. }\end{array}$ & 23 & $\begin{array}{l}\text { B, C, D, E, F, } \\
\text { G. }\end{array}$ & \\
\hline Vermis cerebelar & 24 & $\mathrm{~A}, \mathrm{G}, \mathrm{H}$ & \\
\hline Lobo frontal & 25 & $A, C, E, F$ & \\
\hline Lobo parietal & 26 & $A, C, D, F$ & \\
\hline Lobo temporal & 27 & $\mathrm{~B}, \mathrm{C}, \mathrm{D}$ & \\
\hline Lobo occipital & 28 & $A, C, D, F, G$ & \\
\hline Medula oblonga & 29 & $A, B, C, D$. & \\
\hline Hipocampo & 30 & & J. \\
\hline Amígdala & 31 & & J, L. \\
\hline
\end{tabular}

proporção entre o corpo caloso e o fórnice, este último bem maior em sua coluna e em especial na comissura, logo abaixo do esplênio do corpo caloso (Figura 1E; 2J). Se percebe que o $3^{\circ}$ ventrículo é reduzido, ocorrendo uma continuidade deste com a cavidade do septo pelúcido, esta sim bem desenvolvida. (Figura 2K, J e L). Finalmente, não localizamos os corpos quadrigêmeos ${ }^{21,22}$, ocorrendo duas, em vez de quatro, 
protuberâncias na face dorsal do tronco encefálico, e protuberâncias partindo dos mesmos ligando-os ao tronco encefálico na posição dos braços dos colículos em direção ao núcleo pulvinar do tálamo ${ }^{21}$.

\section{Conclusões}

Concluiu-se que os bulbos olfatórios são de tamanho considerável em relação ao restante do encéfalo, há apenas 2 colículos no tronco encefálico, o fórnice é bem desenvolvido especialmente na sua comissura. A hipófise não se projeta na superfície ventral do encéfalo, permanecendo colada como um disco com seu pedículo rostralmente. A cavidade do septo pelúcido é bem desenvolvida e contínua com o $3^{\circ}$ ventrículo.

\section{Agradecimentos}

Agradecemos ao técnico em laboratório Otavio Cavalcante pelo auxílio com a investigação histológica. Ao professor Carlos Rosemberg Luiz pela disponibilização dos meios técnicos para a realização do trabalho, ambos do Departamento de Morfologia da Universidade Federal de Goiás.

\title{
Contributions to the study of the macroscopy anatomy of Bradypus torquatus (Linnaeus, 1758) and Bradypus variegatus (Schinz, 1825) brain
}

\begin{abstract}
The great preoccupation about the preservation of the south America fauna, have guide researches to a intense productions of the works about them. This work is constitute as a part of this effort to understand the animal structures, in special the gender Bradypus, describing the macroscopic anatomy of the brain in two species, Torquatus and Variegatus, both from the atlantic forest in Brazil. The animals did have a naturally death, were freeze as the standard proceed of the Matinha reserve, the origin of them, and unfreezing, injection, dissection, and colored by the use of appropriate techniques. We observed the neo-cortex formations in a model more primitive than others mammals, a predominance of areas and structures connected to the sense of smell and many differences when compared with other mammals, in especial primates. Among then the occurrence of only two coliculus on the brain trunk, a disk form of the hipophyse, a big fornice, and too development brain peduncles. The circumvolutions are bland and is happen lobos as same to the others mammals with the cerebellum not covered by the projections of the occipital lobos and with proportion until $1 / 4$ in relation to all brain.
\end{abstract}

Key-words Bradypus. Sloth.

\section{Referências}

1 GUEZZI, M. D. et al. Conducto hepatopancreático de la llama (Lama glama). Rev. Chilena de Anatomia, v. 18, n. 1 , p. $27-34,2000$.

2 CARVALHO, M. A. M. et al. Artérias mesentéricas cranial e caudal em cutias (Dasyprocta aguti). Veterinária Notícias, Uberlândia, v. 5, n. 2, p. 17-24, 1999.

3 BLUMENSCHEIN, A. R.; FERREIRA, J. R. Anatomic study of the neotropic primate's submandibular gland ducts. (Cebus apela, Linnaeus, 1766). Rev. Chilena de
Anatomia, v. 20, n. 1, p. 55-61, 2002.

4 OLIVEIRA, A.; FERREIRA, J. R.; BLUMENSCHEIN, A. R. Estudo anatômico do modelo arterial de vasos responsáveis pelo aporte sanguíneo da glândula submandibular de primatas neotropicais (Cebus apella, Linnaeus, 1766). Maringá - Acta Scientiarum. v. 22, n. 2, p. 573-579, 2000.

5 MENEZES, D. J. A. et al. Configuração do sistema venoso portal na cutia (Dasyprocta aguti, Rodentia). Brazilian Journal Vet. Res. Anim. Sci. São Paulo, v. 38, 
n. 6 , p. 263-266, 2001.

6 OLIVEIRA, F. S. et al. Gross anatomical study of the aortic arc branches of the paca (Agouti paca, Linnaeus, 1766). Braz. J. of Vet. Res. and Ani. Sci., São Paulo, v. 38, n. 3, p. 103-105, 2001.

7 MACHADO, G. V. et al. Comportamento anatômico das artérias renais em catetos (Tayassu tacaju, Linnaeus, 1758). Veterinária Notícias, Uberlândia, v. 6, n. 1, p. 17-25, 2000.

8 BONATELLI, M. et al. Análise Morfológica da placenta da paca. Estudo ao microscópio de luz á microscopia eletrônica de transmissão (Agouti paca, Linnaeus, 1766). Braz. Journ. Vet. Res. Anim. Sci. São Paulo, v. 38, n. 5, p. 224-228, 2001.

9 RECKZIEGEL, S. H.; LINDEMANN, T.; CAMPOS, R. A systematic study of the brain base arteries in capybara (Hydrochoerus hydrochaeris). Brazilian Journal of Morphological Sciences, v. 18, n. 2, p. 103-110, 2001.

10 FERREIRA, J. R.; PRAPA, I. L. S. Nomeclatura proposta para denominar as artérias da base do encéfalo do macaco-prego (Cebus apela, Linnaeus, 1766). Acta Scientiarum, Maringá, v. 23, n. 2, p. 635-643, 2001.

11 SIQUEIRA NETO, E. G. B.; FERREIRA, J. R. Estudo anatômico da origem e distribuição dos ramos corticais das artérias cerebrais caudais do encéfalo do macaco prego (Cebus apela, Linnaeus, 1766). Maringá - Acta Scientiarium, v. 24, n. 2, p. 639-646, 2002.

12 SILVA, R.; FERREIRA, J. R. Estudo das artérias cerebelares do macaco prego. Considerações sobre nomenclatura. (Cebus apella, Linnaeus, 1766). Braz. J. Vet. Res. Anim. Sci., São Paulo, v. 39, n. 6, p. 296-300, 2002.

13 GOFFART, M. Function and form in the sloth New York: Pergamon Press, 1971.

14 ANTHONY, J. Mophologie externe du télencéphale dans le genre Bradypus L. (Edentata). Rev. Mammalia, v. 17 , p. 149-163, 1953.

15 BIELICK, P. Constituição do plexus lumbo-sacralis no Bradypus Tridactylus. Ann. Fac. Méd., São Paulo, v. 19, p. 7-11, 1943.

16 HILDEBRAND, M. Análise da estrutura dos vertebrados. São Paulo: Atheneu, 1995.

17 RODRIGUES, H. Técnicas anatômicas. Vitória: [s.n]., 1998.

18 NOMINA ANATÔMICA VETERINÁRIA. International Committee on Veterinary Gross anatomical nomenclature. 4. ed. New York: ICVGAM, 1994.

19 SCHALLER, O. Nomeclatura anatômica veterinária ilustrada. São Paulo: Manole, 1999.

20 JOLLIE, M. Chordate morphology. New York: Publishing Corporation; London: Champman \& Hevl,
1980.

$21 \mathrm{MACHADO}, \mathrm{A}$. Neuroanatomia funcional. Rio de Janeiro: Atheneu, 1988.

22 DYCE, K. M.; SACK, W. O.; WENSING, C. J. G. Tratado de anatomia veterinária. Rio de Janeiro: Guanabara Koogan, 1987.

23 FRANDSON, A.; SPURGEON, B. Anatomia y fisiologia de los animales domésticos. 5. ed. Madrid: Interamericana/Mcgraw-Hill, 1992.

$24 \mathrm{HILL}$, W. C. O. Primates comparative anatomy and taxonomy. IV Cebidae, Part A. Edimburg: University Press, 1960. p. 63-66; 281-282.

25 NUSSHAG, W. Anatomia y fisiologia de los animales domésticos. Zaragoza: Acribia, 1977. 